\title{
YEAST FLORA OF THE MOUTH AND SKIN DURING AND AFTER IRRADIATION FOR ORAL AND LARYNGEAL CANCER
}

\author{
M. V. Martin, U. Al-Tikriti* and P. A. Bramley* \\ Departments of Oral Pathology and *Dental Surgery, School of Clinical Dentistry, \\ University of Sheffield, 31 Claremont Crescent, Sheffield S10 2TA
}

\begin{abstract}
Summary. The quantitative and qualitative changes occurring in the fungal flora of 22 patients with oral and 9 with laryngeal carcinoma were studied during and after radiation therapy. Each patient received 6000 rad of externally applied radiation in divided doses for 5 weeks. The fungal flora was isolated from the patients' oral cavity and irradiated skin sites during irradiation and 2 weeks and 4-6 months afterwards. The number and types of fungi increased in both groups of patients after the start of irradiation and persisted at high levels for at least 4-6 months after treatment. Candida albicans and C. tropicalis were the principal yeasts isolated throughout the period studied but seven other species were also identified. All the yeast isolates were sensitive in vitro to miconazole, ketoconazole, amphotericin B and nystatin. Any of these antifungal agents should be appropriate for therapy.
\end{abstract}

\section{INTRODUCTION}

Radiotherapy administered for the treatment of oral neoplastic disease has profound effects on the oral flora. One of these effects is to cause an increase in the number of yeast colonies that can be isolated from the oral mucosa and saliva (Bodey, 1975). This increase is often associated with some form of superficial fungal infection, which may complicate the radiation therapy (Boggs, Williams and Howell, 1961; Shipman, 1979). These infections are thought to cause severe pain and to be associated with difficulties of swallowing and speech (Beumer, Curtis and Harrison, 1979). Such large numbers of oral yeasts may contribute to the disseminated fungal infections that are often found at post-mortem examination of patients who have had neoplastic disease (Cho and Choi, 1979).

Although fungal infection is known to be associated with radiotherapy, it is difficult for the clinician to anticipate when it will occur in relation to the progress of treatment or how long it will persist; the timing and duration of antifungal therapy is therefore difficult to decide.

Studies on the fungi associated with oral infections during radiotherapy have shown that Candida spp. are usually present, although Aspergillus spp. 
and Torulopsis glabrata are sometimes also isolated (Bodey, 1975). The sensitivity of such yeasts to antifungal drugs is often not known and therapy has to be empirical. Moreover, it is not known whether the sensitivity of yeasts alters during radiation therapy.

Patients receiving externally applied radiotherapy for the treatment of oral and other lesions often suffer side effects at the skin site of irradiation. These usually take the form of dry or moist desquamative lesions accompanied by profound erythema (Moss, Brand and Battifora, 1973). These infections are thought to be predominantly bacterial and the presence of fungi has been reported only occasionally (Walter, Miller and Bomford, 1979).

The aim of the this study was to identify the number and type of fungal colonies isolated from various sites in the mouth from patients receiving radiotherapy for the treatment of oral or laryngeal neoplasm. In addition, we decided to sample the skin sites of irradiation and in a similar manner to monitor any fungal changes. Fungi isolated before, during and after radiotherapy from each site were tested against three commonly used and one new antifungal agent: nystatin, amphotericin B, miconazole and ketoconazole.

\section{PATIENTS AND METHODS}

Patients and samples. Thirty-one patients were studied, 22 with oral neoplasm (the "oral group") and 9 with laryngeal neoplasm (the "laryngeal group"). The patients were selected from those attending for either inpatient or outpatient treatment at Weston Park Hospital, Sheffield. It was planned that the groups would be age and sex matched, but enough patients for precise matching could not be found. The oral group ( 18 males, 4 females) had an average age of 63 years (range $51-79$ ). The neoplastic lesions being treated in this group were 21 squamous-cell carcinomas and one muco-epidermoid carcinoma. The squamous-cell carcinomas involved the tongue (5), floor of mouth (4), palate and antrum (9), submandibular gland (1) and tonsillar fossa (2). The muco-epidermoid carcinoma involved the floor of the mouth and submandibular gland. The laryngeal group ( 7 males, 2 females) had an average age of 58 years (range 43-70). The lesions involved the vocal cords (4), epiglottis (4) and pyriform fossa (1) and were all squamous-cell carcinomas.

Each patient received a total of $6000 \mathrm{rad}$ at a beam distance of $100 \mathrm{~cm}$ and a field width of $7-10 \mathrm{~cm}$. Twenty-nine patients ( 22 oral, 7 laryngeal) were treated with the linear accelerator set at a potential of $8 \mathrm{MV}$ and two patients were treated with cobalt 60 . Radiation was applied to alternate sides in 25 sessions during 5 weeks. Each patient was provided with the saliva substitute described by Shannon, John and Starcke (1978) ad libitum and aspirin as an analgesic when required; antibacterial or antifungal agents were not given. None of the patients had had previous surgery or any other treatment for their neoplastic lesions.

Each patient was examined before treatment and thereafter at the end of 1,2,3,4 and 5 weeks of radiotherapy and 2 weeks after it ended. A final examination was made 4-6 months after completion of radiotherapy. At each examination plain cottonwool swabs were used to sample, by a standardised method, the mucosa of the palate, dorsum of tongue, floor of mouth in the region of the molar teeth, upper and lower buccal sulci, and the skin sites of irradiation.

Microbiological methods. Swabs were inoculated onto Sabouraud's agar (Lab M, London) within $2 \mathrm{~h}$ of sampling by a standardised technique; plates were incubated at $30^{\circ} \mathrm{C}$ for $72 \mathrm{~h}$. The fungal colonies were counted on every plate and scored 0 for no colonies, 1 for $1-5$ colonies, 2 for 6-15 and 3 in excess of fifteen. The total score for the patients in each group at every examination time was then calculated as a percentage of the maximal score possible. The percentage scores obtained for the oral and the laryngeal groups were then plotted against time in weeks since the start of the treatment. The different colony types from all the plates were subcultured onto Sabouraud's agar and identified by the methods of Dolan (1971) and the 
sugar-assimilation tests of Martin and Schneidau (1970). Each colony identified was then subcultured onto the yeast-nitrogen-base (YNB) agar described by Shadomy (1969).

Sensitivity tests. Inocula for sensitivity testing were harvested from overnight growths at $30^{\circ} \mathrm{C}$ on YNB medium and adjusted to $c .5 \times 10^{6} / \mathrm{ml}$ in fresh YNB medium; $0.2 \mathrm{ml}$ of this suspension was used for inoculation. The suspension of the individual organism was then spread evenly over the surface of YNB-agar plates containing either miconazole or ketoconazole $0 \cdot 1-50 \mu \mathrm{g} / \mathrm{ml}$, or amphotericin B $0 \cdot 1-10 \mu \mathrm{g} / \mathrm{ml}$ or nystatin $1-30 \mathrm{units} / \mathrm{ml}$. The amphotericin B was prepared from that used for intravenous injection diluted with sterile distilled water. Miconazole and ketoconazole were dissolved in dimethylsulphoxide to a concentration of 50 $\mu \mathrm{g} / \mathrm{ml}$ and then further diluted with water. Nystatin, 5050 units $/ \mathrm{mg}$, was dissolved in dimethylformamide to a concentration of $1000 \mathrm{units} / \mathrm{ml}$ and then diluted with water as required. The inoculated sensitivity plates were incubated at $30^{\circ} \mathrm{C}$ for $72 \mathrm{~h}$ and the minimum inhibitory concentration (MIC) for each drug was recorded as the lowest concentration of the antifungal agent that completely inhibited visible growth. In every set of determinations, one plate per strain to which antifungal agent had not been added, was included to ensure that growth had occurred. Laboratory strains of Saccharomyces cerevisiae, C. albicans, C. tropicalis and $T$. glabrata, for which the MICs of the antifungal agents were known, were included in every set of determinations.

\section{RESULTS}

\section{Quantitative changes in yeast flora}

The majority of patients with either oral or laryngeal cancer yielded fungi from one or more oral sites before treatment (fig. 1), with less than five colonies being isolated from each site. In contrast, fungi were not isolated from the skin sites of irradiation before treatment in either group of patients (fig. 2). After the start of treatment the fungal isolations from the mouth in both

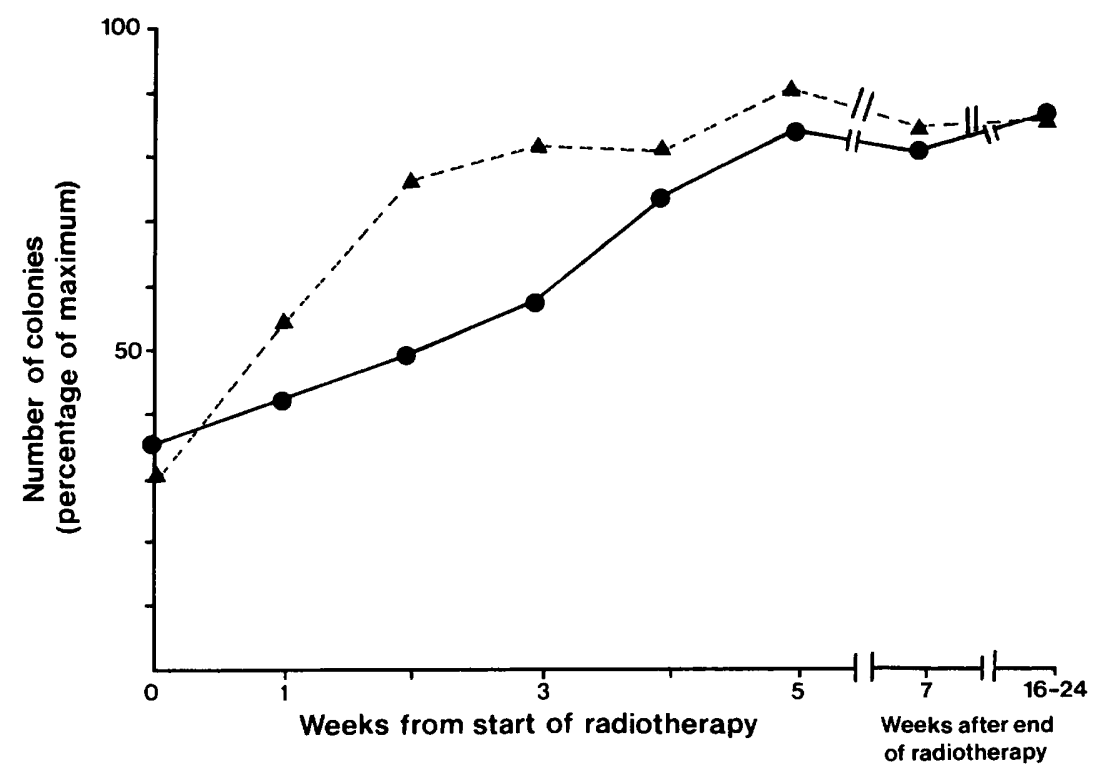

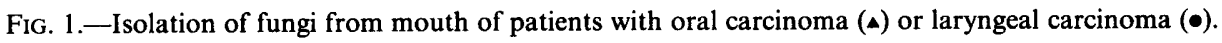




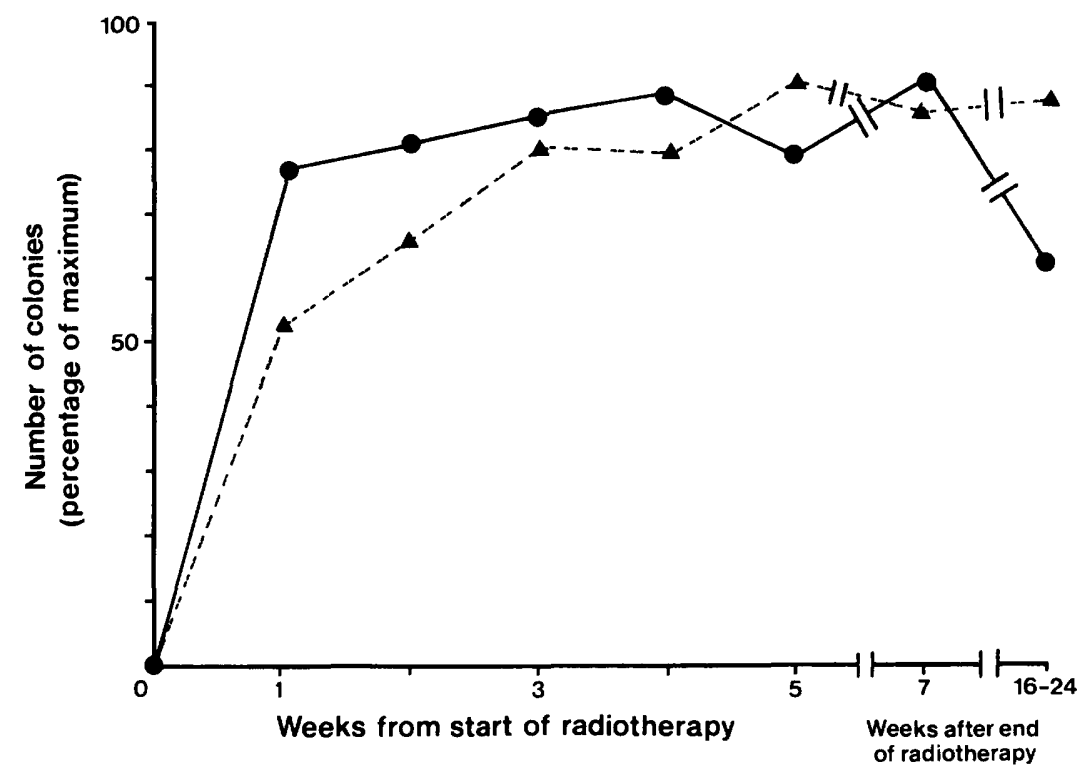

Fig. 2.-Isolation of fungi from skin sites of irradiation of patients with oral carcinoma $(\Delta)$ or laryngeal carcinoma (•).

groups rose rapidly, the rise being faster in the oral group (fig. 1). By the end of the 4 th week of treatment the majority of patients had grade- 3 scores. After the end of radiotherapy (week 5) the fungal isolations did not fall but remained high in both groups; 4-6 months later little diminution in fungal isolations was seen.

Fungal isolation from the skin sites of irradiation showed a similar pattern for both groups (fig. 2). After the start of treatment there was an initial and rapid rise in the number of fungi, which by the 3rd week of treatment was approaching maximal values. Six months after the end of treatment a slight

TABLE I

Distribution of fungal species among sites of patients in the laryngeal group

\begin{tabular}{|c|c|c|c|c|c|c|c|c|}
\hline \multirow[b]{3}{*}{ Species } & \multicolumn{6}{|c|}{ Number of isolates from } & \multirow{3}{*}{\multicolumn{2}{|c|}{ Total $\begin{array}{c}\text { Percentage } \\
\text { of all } \\
\text { isolates }\end{array}$}} \\
\hline & \multirow[b]{2}{*}{ palate } & \multirow[b]{2}{*}{ tongue } & \multirow{2}{*}{$\begin{array}{l}\text { floor of } \\
\text { mouth }\end{array}$} & \multicolumn{2}{|c|}{ buccal sulcus } & \multirow[b]{2}{*}{ skin } & & \\
\hline & & & & upper & lower & & & \\
\hline Candida albicans & 51 & 37 & 46 & 41 & 37 & 44 & 256 & $59 \cdot 3$ \\
\hline C. tropicalis & 13 & 16 & 5 & 19 & 14 & 7 & 74 & $17 \cdot 1$ \\
\hline C. pseudotropicalis & 1 & 1 & 3 & 0 & 2 & 0 & 7 & 1.6 \\
\hline C. guilliermondi & 3 & 7 & 3 & 1 & $\overline{4}$ & 0 & 18 & $4 \cdot 2$ \\
\hline C. parapsilosis & 1 & 2 & 1 & 2 & 1 & 1 & 8 & $1 . \overline{9}$ \\
\hline C. mycoderma & 0 & 0 & 2 & 3 & 0 & 3 & 8 & 1.9 \\
\hline Torulopsis glabrata & 0 & 1 & 2 & 0 & 6 & 12 & 21 & 4.9 \\
\hline Saccharomyces spp. & 1 & 8 & 7 & 6 & 4 & 2 & 28 & $6 \cdot 5$ \\
\hline Rhodotorula rubra & 0 & 0 & 0 & 0 & 1 & 0 & 1 & $0 \cdot 2$ \\
\hline Unidentified or not subcultured & 2 & 0 & 3 & 0 & 3 & 3 & 11 & $2 \cdot \overline{5}$ \\
\hline
\end{tabular}


decrease was noted in the isolations from the laryngeal group but the oral group still had almost maximal fungal scores.

\section{Qualitative changes in yeast flora}

At the first examination the majority of isolates ( $>96 \%$ ) were C. albicans; occasionally $C$. tropicalis and $C$. krusei were identified. After 1 week of treatment the number of different isolates rose in both groups from all sites and remained varied for at least 4-6 months after treatment was complete. Tables I and II show the yeast flora for each site from each group. From the laryngeal group of patients, 432 isolates were identified; $C$. albicans and C. tropicalis accounted for $76.4 \%$ of them (table I). Four other species of Candida were identified together with $T$. glabrata and Rhodotorula rubra. Three isolates

TABLE II

Distribution of fungal species among sites of patients in the oral group

\begin{tabular}{|c|c|c|c|c|c|c|c|c|}
\hline \multirow[b]{3}{*}{ Species } & \multicolumn{6}{|c|}{ Number of isolates from } & & \multirow{3}{*}{$\begin{array}{c}\text { Percentage } \\
\text { of all } \\
\text { isolates }\end{array}$} \\
\hline & \multirow[b]{2}{*}{ palate } & \multirow[b]{2}{*}{ tongue } & \multirow{2}{*}{$\begin{array}{c}\text { floor of } \\
\text { mouth }\end{array}$} & \multicolumn{2}{|c|}{ buccal sulcus } & \multirow[b]{2}{*}{ skin } & \multirow[b]{2}{*}{ Total } & \\
\hline & & & & upper & lower & & & \\
\hline C. albicans & 103 & 123 & 109 & 134 & 143 & 59 & 671 & $65 \cdot 8$ \\
\hline C. tropicalis & 41 & 26 & 36 & 18 & 12 & 31 & 164 & $16 \cdot 1$ \\
\hline C. pseudotropicalis & 3 & 0 & 8 & 7 & 4 & 7 & 29 & $2 \cdot 8$ \\
\hline C. guilliermondi & 7 & 3 & 1 & 2 & 0 & 1 & 14 & $1 \cdot 4$ \\
\hline C. parapsilosis & 1 & 6 & 2 & 0 & 1 & 1 & 11 & $1 \cdot 1$ \\
\hline C. krusei & 12 & 2 & 1 & 0 & 3 & 1 & 19 & 1.9 \\
\hline C. mycoderma & 0 & 1 & 0 & 0 & 1 & 21 & 23 & $2 \cdot 3$ \\
\hline T. glabrata & 1 & 0 & 2 & 3 & 4 & 14 & 24 & $2 \cdot 4$ \\
\hline Saccharomyces spp. & 1 & 8 & 10 & 2 & 0 & 18 & 39 & $3 \cdot 8$ \\
\hline R. rubra & 0 & 1 & 0 & 3 & 1 & 14 & 19 & 1.9 \\
\hline Unidentified & 1 & 0 & 1 & 1 & 1 & 3 & 7 & 0.7 \\
\hline
\end{tabular}

failed to grow after repeated subculture; they were yeast-like on gram-stained films. Eight other organisms that remained unidentified were Candida spp. but distinction between $C$. albicans and $C$. stellatoidea could not be achieved because of variability of the results of repeated fermentation and assimilation tests.

A similar distribution of isolates was seen in the oral group of patients, although more (1020) were identified (table II). The reason for this was a greater variety in their colonial appearance. C. albicans and C. tropicalis again accounted for the majority of isolates. C. krusei was also isolated from this group but not from patients in the laryngeal group. In addition, $C$. mycoderma was isolated predominantly from the skin sites.

From both groups of patients 87 samples (63 oral, 24 laryngeal) yielded growths of Klebsiella species on Sabouraud's agar. They were identified, by the methods of Cowan (1974), as mixtures of Klebsiella aerogenes, $K$. pneumoniae and, from four samples, $K$. oxytoca. 
TABLE III

Minimum inhibitory concentration of nystatin for yeast isolates

\begin{tabular}{|c|c|c|c|c|c|c|c|c|c|c|c|c|c|}
\hline \multirow[b]{2}{*}{ Species } & \multirow{2}{*}{$\begin{array}{c}\text { Group } \\
\text { derivation }\end{array}$} & \multirow{2}{*}{$\begin{array}{c}\text { Number } \\
\text { tested }\end{array}$} & \multicolumn{11}{|c|}{$\begin{array}{l}\text { Number of isolates inhibited by nystatin at concen- } \\
\text { tration (units/ml) of }\end{array}$} \\
\hline & & & 1 & 2 & 3 & 4 & 5 & $7 \cdot 5$ & 10 & 15 & 20 & 25 & 30 \\
\hline C. albicans & $\begin{array}{l}\mathrm{O} \\
\mathrm{L}\end{array}$ & $\begin{array}{l}671 \\
256\end{array}$ & $\dddot{1}$ & $\begin{array}{c}1 \\
\ldots\end{array}$ & $\begin{array}{l}48 \\
22\end{array}$ & $\begin{array}{l}2 \\
4\end{array}$ & $\begin{array}{l}52 \\
83\end{array}$ & $\begin{array}{r}116 \\
5\end{array}$ & $\begin{array}{l}433 \\
136\end{array}$ & $\begin{array}{l}11 \\
\ldots\end{array}$ & $\begin{array}{l}8 \\
5\end{array}$ & $\begin{array}{l}\ldots \\
\ldots\end{array}$ & $\begin{array}{l}\ldots \\
\ldots\end{array}$ \\
\hline C. tropicalis & $\begin{array}{l}\mathrm{O} \\
\mathrm{L}\end{array}$ & $\begin{array}{r}164 \\
74\end{array}$ & $\cdots$ & $\begin{array}{l}\ldots \\
\ldots\end{array}$ & $\begin{array}{l}1 \\
1\end{array}$ & $\dddot{1}$ & 11 & $\begin{array}{r}10 \\
8\end{array}$ & 11 & $\begin{array}{l}83 \\
27\end{array}$ & $\begin{array}{r}61 \\
4\end{array}$ & $\begin{array}{r}8 \\
11\end{array}$ & $\begin{array}{l}\ldots \\
\cdots\end{array}$ \\
\hline C. pseudotropicalis & $\begin{array}{l}\mathrm{O} \\
\mathrm{L}\end{array}$ & $\begin{array}{r}29 \\
7\end{array}$ & $\begin{array}{l}\ldots \\
\cdots\end{array}$ & $\begin{array}{c}1 \\
\ldots\end{array}$ & $\begin{array}{r}1 \\
\ldots\end{array}$ & $\begin{array}{l}\ldots \\
\ldots\end{array}$ & $\begin{array}{l}2 \\
3\end{array}$ & $\dddot{1}$ & $\begin{array}{c}4 \\
\ldots\end{array}$ & $\begin{array}{r}21 \\
2\end{array}$ & $\dddot{i}$ & $\begin{array}{l}\ldots \\
\ldots\end{array}$ & $\begin{array}{l}\ldots \\
\ldots\end{array}$ \\
\hline C. guilliermondi & $\begin{array}{l}\mathrm{O} \\
\mathrm{L}\end{array}$ & $\begin{array}{l}14 \\
18\end{array}$ & $\begin{array}{l}8 \\
6\end{array}$ & $\begin{array}{l}1 \\
6\end{array}$ & $\begin{array}{l}1 \\
3\end{array}$ & $\begin{array}{l}4 \\
2\end{array}$ & $\dddot{i}$ & $\begin{array}{l}\cdots \\
\cdots\end{array}$ & $\begin{array}{l}\ldots \\
\ldots\end{array}$ & $\begin{array}{l}\ldots \\
\ldots\end{array}$ & $\begin{array}{l}\ldots \\
\cdots\end{array}$ & $\begin{array}{l}\ldots \\
\ldots\end{array}$ & $\begin{array}{l}\ldots \\
\ldots\end{array}$ \\
\hline C. parapsilosis & $\begin{array}{l}\mathrm{O} \\
\mathrm{L}\end{array}$ & $\begin{array}{r}11 \\
8\end{array}$ & $\ldots$ & $\begin{array}{l}\ldots \\
\ldots\end{array}$ & $\begin{array}{l}\ldots \\
\cdots\end{array}$ & $\begin{array}{l}\ldots \\
\cdots\end{array}$ & $\begin{array}{l}\ldots \\
\cdots\end{array}$ & $\dddot{2}$ & $\begin{array}{l}4 \\
1\end{array}$ & $\begin{array}{l}4 \\
2\end{array}$ & $\begin{array}{l}3 \\
3\end{array}$ & $\begin{array}{l}\ldots \\
\ldots\end{array}$ & $\begin{array}{l}\ldots \\
\ldots\end{array}$ \\
\hline C. krusei & $\begin{array}{l}\mathrm{O} \\
\mathrm{L}\end{array}$ & $\begin{array}{r}19 \\
0\end{array}$ & $\cdots$ & $\begin{array}{l}\cdots \\
\cdots\end{array}$ & $\begin{array}{l}\ldots \\
\ldots\end{array}$ & $\begin{array}{l}\cdots \\
\cdots\end{array}$ & $\begin{array}{l}\cdots \\
\cdots\end{array}$ & $\cdots$ & $\begin{array}{l}\cdots \\
\cdots\end{array}$ & $\begin{array}{c}7 \\
\ldots\end{array}$ & $\ldots$ & $\begin{array}{r}4 \\
\ldots\end{array}$ & $\begin{array}{c}8 \\
\cdots\end{array}$ \\
\hline C. mycoderma & $\begin{array}{l}\mathrm{O} \\
\mathrm{L}\end{array}$ & $\begin{array}{r}23 \\
8\end{array}$ & $\ldots$ & $\begin{array}{l}\ldots \\
\cdots\end{array}$ & $\begin{array}{l}1 \\
2\end{array}$ & $\begin{array}{l}\ldots \\
\ldots\end{array}$ & $\dddot{2}$ & $\dddot{1}$ & $\begin{array}{l}6 \\
1\end{array}$ & $\begin{array}{r}16 \\
2\end{array}$ & $\begin{array}{l}\ldots \\
\ldots\end{array}$ & $\begin{array}{l}\ldots \\
\ldots\end{array}$ & $\begin{array}{l}\ldots \\
\ldots\end{array}$ \\
\hline$T$. glabrata & $\begin{array}{l}\mathrm{O} \\
\mathrm{L}\end{array}$ & $\begin{array}{l}24 \\
21\end{array}$ & $\ldots$ & $\begin{array}{r}13 \\
4\end{array}$ & $\begin{array}{l}\ldots \\
\ldots\end{array}$ & $\begin{array}{r}4 \\
11\end{array}$ & $\begin{array}{l}\ldots \\
\ldots\end{array}$ & $\dddot{3}$ & $\begin{array}{l}1 \\
3\end{array}$ & 5 & $\begin{array}{c}1 \\
\ldots\end{array}$ & $\begin{array}{l}\ldots \\
\ldots\end{array}$ & $\begin{array}{l}\ldots \\
\ldots\end{array}$ \\
\hline R. rubra & $\begin{array}{l}\mathrm{O} \\
\mathrm{L}\end{array}$ & $\begin{array}{r}19 \\
1\end{array}$ & $\cdots$ & $\begin{array}{l}\cdots \\
\cdots\end{array}$ & $\begin{array}{l}\ldots \\
\cdots\end{array}$ & $\begin{array}{r}3 \\
\cdots\end{array}$ & $\begin{array}{c}2 \\
\cdots\end{array}$ & $\begin{array}{l}6 \\
1\end{array}$ & $\begin{array}{l}8 \\
\cdots\end{array}$ & $\begin{array}{l}\ldots \\
\cdots\end{array}$ & $\begin{array}{l}\cdots \\
\cdots\end{array}$ & $\begin{array}{l}\ldots \\
\cdots\end{array}$ & $\begin{array}{l}\ldots \\
\ldots\end{array}$ \\
\hline
\end{tabular}

$\mathrm{O}=$ patients of the oral group; $\mathrm{L}=$ patients of the laryngeal group.

TABLE IV

Minimum inhibitory concentration of amphotericin $B$ for yeast isolates

\begin{tabular}{|c|c|c|c|c|c|c|c|c|c|}
\hline \multirow[b]{2}{*}{ Species } & \multirow{2}{*}{$\begin{array}{c}\text { Group } \\
\text { derivation }\end{array}$} & \multirow{2}{*}{$\begin{array}{c}\text { Number } \\
\text { tested }\end{array}$} & \multicolumn{7}{|c|}{$\begin{array}{l}\text { Number of isolates inhibited } \\
\text { by amphotericin } \mathrm{B} \\
\text { at a concentration }(\mu \mathrm{g} / \mathrm{ml}) \text { of }\end{array}$} \\
\hline & & & $0 \cdot 1$ & 0.5 & 1.0 & 2 & 4 & 5 & 10 \\
\hline C. albicans & $\begin{array}{l}\mathrm{O} \\
\mathrm{L}\end{array}$ & $\begin{array}{l}671 \\
256\end{array}$ & $\begin{array}{r}121 \\
83\end{array}$ & $\begin{array}{l}526 \\
141\end{array}$ & $\begin{array}{l}13 \\
32\end{array}$ & $\begin{array}{c}8 \\
\cdots\end{array}$ & $\begin{array}{c}3 \\
\cdots\end{array}$ & $\begin{array}{l}\ldots \\
\cdots\end{array}$ & $\begin{array}{l}\ldots \\
\cdots\end{array}$ \\
\hline C. tropicalis & $\begin{array}{l}\mathrm{O} \\
\mathrm{L}\end{array}$ & $\begin{array}{r}164 \\
74\end{array}$ & $\begin{array}{r}3 \\
19\end{array}$ & $\begin{array}{l}23 \\
31\end{array}$ & $\begin{array}{r}101 \\
11\end{array}$ & $\begin{array}{l}23 \\
10\end{array}$ & $\begin{array}{l}8 \\
2\end{array}$ & $\begin{array}{l}6 \\
1\end{array}$ & $\begin{array}{l}\cdots \\
\cdots\end{array}$ \\
\hline C. pseudotropicalis & $\begin{array}{l}\mathrm{O} \\
\mathrm{L}\end{array}$ & $\begin{array}{r}29 \\
7\end{array}$ & $\dddot{i}$ & $\begin{array}{r}10 \\
3\end{array}$ & $\begin{array}{r}18 \\
3\end{array}$ & $\begin{array}{r}1 \\
\ldots\end{array}$ & $\begin{array}{l}\ldots \\
\ldots\end{array}$ & $\begin{array}{l}\ldots \\
\ldots\end{array}$ & $\begin{array}{l}\ldots \\
\ldots\end{array}$ \\
\hline C. guilliermondi & $\begin{array}{l}\mathrm{O} \\
\mathrm{L}\end{array}$ & $\begin{array}{l}14 \\
18\end{array}$ & $\begin{array}{r}8 \\
11\end{array}$ & $\begin{array}{l}3 \\
6\end{array}$ & $\begin{array}{l}3 \\
1\end{array}$ & $\begin{array}{l}\ldots \\
\ldots\end{array}$ & $\begin{array}{l}\ldots \\
\ldots\end{array}$ & $\begin{array}{l}\ldots \\
\ldots\end{array}$ & $\begin{array}{l}\ldots \\
\cdots\end{array}$ \\
\hline C. parapsilosis & $\underset{\mathrm{L}}{\mathrm{O}}$ & $\begin{array}{r}11 \\
8\end{array}$ & $\begin{array}{c}4 \\
\ldots\end{array}$ & $\begin{array}{l}\ldots \\
\ldots\end{array}$ & $\begin{array}{l}2 \\
3\end{array}$ & $\dddot{2}$ & $\begin{array}{l}1 \\
3\end{array}$ & $\begin{array}{l}4 \\
\ldots\end{array}$ & $\begin{array}{l}\ldots \\
\cdots\end{array}$ \\
\hline C. krusei & $\begin{array}{l}\mathrm{O} \\
\mathrm{L}\end{array}$ & $\begin{array}{r}19 \\
0\end{array}$ & $\begin{array}{l}\ldots \\
\cdots\end{array}$ & $\begin{array}{r}4 \\
\ldots\end{array}$ & $\begin{array}{r}4 \\
\ldots\end{array}$ & $\begin{array}{r}4 \\
\ldots\end{array}$ & $\begin{array}{r}3 \\
\ldots\end{array}$ & $\begin{array}{r}4 \\
\ldots\end{array}$ & $\begin{array}{l}\ldots \\
\ldots\end{array}$ \\
\hline C. mycoderma & $\begin{array}{l}\mathrm{O} \\
\mathrm{L}\end{array}$ & $\begin{array}{r}23 \\
8\end{array}$ & $\begin{array}{l}3 \\
7\end{array}$ & $\begin{array}{l}16 \\
\cdots\end{array}$ & $\begin{array}{l}2 \\
1\end{array}$ & $\begin{array}{c}2 \\
\cdots\end{array}$ & $\begin{array}{l}\cdots \\
\cdots\end{array}$ & $\begin{array}{l}\cdots \\
\cdots\end{array}$ & $\begin{array}{l}\ldots \\
\cdots\end{array}$ \\
\hline T. glabrata & $\begin{array}{l}\text { O } \\
\mathrm{L}\end{array}$ & $\begin{array}{l}24 \\
21\end{array}$ & $\dddot{4}$ & $\dddot{7}$ & $\begin{array}{l}\cdots \\
\cdots\end{array}$ & $\begin{array}{r}16 \\
7\end{array}$ & $\begin{array}{l}4 \\
3\end{array}$ & $\begin{array}{c}4 \\
\ldots\end{array}$ & $\begin{array}{l}\cdots \\
\cdots\end{array}$ \\
\hline R. rubra & $\begin{array}{l}\mathrm{O} \\
\mathrm{L}\end{array}$ & $\begin{array}{r}19 \\
1\end{array}$ & $\begin{array}{l}11 \\
\ldots\end{array}$ & $\begin{array}{l}4 \\
1\end{array}$ & $\begin{array}{r}4 \\
\ldots\end{array}$ & $\begin{array}{l}\ldots \\
\cdots\end{array}$ & $\begin{array}{l}\ldots \\
\ldots\end{array}$ & $\begin{array}{l}\ldots \\
\cdots\end{array}$ & $\begin{array}{l}\ldots \\
\cdots\end{array}$ \\
\hline
\end{tabular}

Abbreviations as in table III. 


\section{Sensitivity tests}

The results of the sensitivity tests with the four antifungal agents are shown in tables II-VI. The MICs of all the isolates to nystatin (table III) ranged from 1 to 30 units $/ \mathrm{ml}$. The highest MICs were recorded for $C$. krusei and $C$. tropicalis, whilst the $C$. albicans strains were all sensitive in the range 1-20 units $/ \mathrm{ml}$. On 23 occasions, separate isolates from the same site were found to differ by at least threefold in sensitivity to nystatin.

All the isolates were very sensitive to amphotericin B (table IV), the highest MIC being $5 \mu \mathrm{g} / \mathrm{ml}$; more than $90 \%$ of the isolates had an MIC of $1 \mu \mathrm{g} / \mathrm{ml}$ or less.

TABLE V

Minimum inhibitory concentration of miconazole for yeast isolates

\begin{tabular}{|c|c|c|c|c|c|c|c|c|c|c|c|c|c|c|c|}
\hline \multirow[b]{2}{*}{ Species } & \multirow{2}{*}{$\begin{array}{l}\text { Group } \\
\text { derivation }\end{array}$} & \multirow{2}{*}{$\begin{array}{c}\text { Number } \\
\text { tested }\end{array}$} & \multicolumn{13}{|c|}{$\begin{array}{l}\text { Number of isolates inhibited by miconazole at } \\
\text { concentration }(\mu \mathrm{g} / \mathrm{ml}) \text { of }\end{array}$} \\
\hline & & & $0 \cdot 1$ & 0.5 & 1.0 & $2 \cdot 0$ & $4 \cdot 0$ & 5.0 & 10 & 15 & 20 & 25 & 30 & 40 & 50 \\
\hline C. albicans & $\begin{array}{l}\mathrm{O} \\
\mathrm{L}\end{array}$ & $\begin{array}{l}671 \\
256\end{array}$ & $\begin{array}{l}94 \\
85\end{array}$ & $\begin{array}{l}63 \\
42\end{array}$ & $\begin{array}{r}111 \\
48\end{array}$ & $\begin{array}{l}67 \\
61\end{array}$ & $\begin{array}{r}185 \\
19\end{array}$ & $\begin{array}{l}95 \\
\ldots\end{array}$ & $\begin{array}{r}54 \\
1\end{array}$ & $\begin{array}{l}\cdots \\
\cdots\end{array}$ & $\begin{array}{l}1 \\
\cdots\end{array}$ & 1 & $\begin{array}{l}\cdots \\
\cdots\end{array}$ & $\begin{array}{l}\ldots \\
\cdots\end{array}$ & $\begin{array}{l}\cdots \\
\cdots\end{array}$ \\
\hline C. tropicalis & $\begin{array}{l}\mathrm{O} \\
\mathrm{L}\end{array}$ & $\begin{array}{r}164 \\
74\end{array}$ & $\begin{array}{c}8 \\
\cdots\end{array}$ & $\begin{array}{l}4 \\
9\end{array}$ & $\begin{array}{c}9 \\
\cdots\end{array}$ & $\begin{array}{r}23 \\
7\end{array}$ & 11 & $\begin{array}{l}4 \\
1\end{array}$ & $\begin{array}{r}8 \\
21\end{array}$ & $\begin{array}{l}42 \\
18\end{array}$ & $\begin{array}{r}31 \\
1\end{array}$ & $\begin{array}{r}18 \\
1\end{array}$ & $\begin{array}{l}5 \\
2\end{array}$ & $\begin{array}{c}1 \\
\cdots\end{array}$ & $\begin{array}{l}\ldots \\
\ldots\end{array}$ \\
\hline C.pseudotropicalis & $\begin{array}{l}\text { O } \\
\text { L }\end{array}$ & $\begin{array}{r}29 \\
7\end{array}$ & $\begin{array}{r}1 \\
\ldots\end{array}$ & $\begin{array}{l}1 \\
1\end{array}$ & $\begin{array}{l}3 \\
2\end{array}$ & 6 & $\begin{array}{l}4 \\
4\end{array}$ & $\begin{array}{l}\ldots \\
\ldots\end{array}$ & $\begin{array}{l}14 \\
\ldots\end{array}$ & $\begin{array}{l}\ldots \\
\ldots\end{array}$ & $\begin{array}{l}\ldots \\
\ldots\end{array}$ & $\begin{array}{l}\ldots \\
\ldots\end{array}$ & $\begin{array}{l}\ldots \\
\ldots\end{array}$ & $\begin{array}{l}\ldots \\
\ldots\end{array}$ & $\begin{array}{l}\ldots \\
\ldots\end{array}$ \\
\hline C. guilliermondi & $\begin{array}{l}\mathrm{O} \\
\mathrm{L}\end{array}$ & $\begin{array}{l}14 \\
18\end{array}$ & $\ddot{i}$ & $\begin{array}{l}2 \\
1\end{array}$ & $\begin{array}{l}2 \\
3\end{array}$ & $\begin{array}{l}2 \\
4\end{array}$ & $\begin{array}{l}4 \\
6\end{array}$ & $\begin{array}{l}1 \\
3\end{array}$ & $\begin{array}{r}3 \\
\ldots\end{array}$ & $\begin{array}{l}\ldots \\
\ldots\end{array}$ & $\begin{array}{l}\ldots \\
\ldots\end{array}$ & $\begin{array}{l}\ldots \\
\ldots\end{array}$ & $\begin{array}{l}\ldots \\
\ldots\end{array}$ & $\begin{array}{l}\ldots \\
\ldots\end{array}$ & $\begin{array}{l}\ldots \\
\ldots\end{array}$ \\
\hline C. parapsilosis & $\begin{array}{l}\mathrm{O} \\
\mathrm{L}\end{array}$ & $\begin{array}{r}11 \\
8\end{array}$ & 2 & $\begin{array}{l}4 \\
4\end{array}$ & $\begin{array}{l}4 \\
1\end{array}$ & $\begin{array}{l}3 \\
1\end{array}$ & $\begin{array}{l}\ldots \\
\ldots\end{array}$ & $\begin{array}{l}\ldots \\
\ldots\end{array}$ & $\begin{array}{l}\ldots \\
\ldots\end{array}$ & $\begin{array}{l}\ldots \\
\ldots\end{array}$ & $\ldots$ & $\begin{array}{l}\ldots \\
\ldots\end{array}$ & $\begin{array}{l}\ldots \\
\ldots\end{array}$ & $\begin{array}{l}\ldots \\
\ldots\end{array}$ & $\begin{array}{l}\ldots \\
\ldots\end{array}$ \\
\hline C. krusei & $\begin{array}{l}\mathrm{O} \\
\mathrm{L}\end{array}$ & $\begin{array}{r}19 \\
0\end{array}$ & $\begin{array}{c}2 \\
\ldots\end{array}$ & $\begin{array}{l}10 \\
\ldots\end{array}$ & $\begin{array}{c}4 \\
\ldots\end{array}$ & $\begin{array}{c}3 \\
\ldots\end{array}$ & $\begin{array}{l}\ldots \\
\ldots\end{array}$ & $\begin{array}{l}\ldots \\
\ldots\end{array}$ & $\begin{array}{l}\ldots \\
\ldots\end{array}$ & $\begin{array}{l}\ldots \\
\ldots\end{array}$ & $\begin{array}{l}\ldots \\
\ldots\end{array}$ & $\begin{array}{l}\ldots \\
\ldots\end{array}$ & $\begin{array}{l}\ldots \\
\ldots\end{array}$ & $\begin{array}{l}\ldots \\
\ldots\end{array}$ & $\begin{array}{l}\ldots \\
\ldots\end{array}$ \\
\hline C. mycoderma & $\begin{array}{l}\mathrm{O} \\
\mathrm{L}\end{array}$ & $\begin{array}{r}23 \\
8\end{array}$ & $\begin{array}{c}4 \\
\ldots\end{array}$ & $\begin{array}{r}4 \\
\ldots\end{array}$ & $\begin{array}{r}11 \\
2\end{array}$ & $\begin{array}{l}4 \\
4\end{array}$ & $\dddot{2}$ & $\begin{array}{l}\ldots \\
\ldots\end{array}$ & $\begin{array}{l}\ldots \\
\ldots\end{array}$ & $\begin{array}{l}\ldots \\
\ldots\end{array}$ & $\begin{array}{l}\ldots \\
\ldots\end{array}$ & $\begin{array}{l}\ldots \\
\ldots\end{array}$ & $\begin{array}{l}\ldots \\
\ldots\end{array}$ & $\begin{array}{l}\ldots \\
\ldots\end{array}$ & $\begin{array}{l}\ldots \\
\ldots\end{array}$ \\
\hline T. glabrata & $\begin{array}{l}\mathrm{O} \\
\mathrm{L}\end{array}$ & $\begin{array}{l}24 \\
21\end{array}$ & $\begin{array}{l}\ldots \\
\ldots \\
\end{array}$ & $\begin{array}{l}\ldots \\
\ldots\end{array}$ & $\begin{array}{l}\ldots \\
\ldots\end{array}$ & $\begin{array}{l}\ldots \\
\ldots\end{array}$ & $\ldots$ & $\begin{array}{l}3 \\
1\end{array}$ & $\begin{array}{r}11 \\
6\end{array}$ & $\begin{array}{l}8 \\
3\end{array}$ & $\begin{array}{l}1 \\
7\end{array}$ & $\begin{array}{l}\ldots \\
\ldots\end{array}$ & $\begin{array}{l}\ldots \\
\ldots\end{array}$ & $\begin{array}{c}1 \\
\ldots\end{array}$ & $\begin{array}{l}\ldots \\
\ldots\end{array}$ \\
\hline R. rubra & $\begin{array}{l}\mathrm{O} \\
\mathrm{L}\end{array}$ & $\begin{array}{r}19 \\
1\end{array}$ & $\begin{array}{l} \\
\ldots \\
\ldots\end{array}$ & $\begin{array}{c}4 \\
\ldots\end{array}$ & $\begin{array}{l}7 \\
1\end{array}$ & $\begin{array}{r}6 \\
\ldots\end{array}$ & $\begin{array}{c}2 \\
\ldots\end{array}$ & $\begin{array}{l}\ldots \\
\ldots\end{array}$ & $\begin{array}{l}\ldots \\
\ldots\end{array}$ & $\begin{array}{l}\ldots \\
\ldots\end{array}$ & $\begin{array}{l}\ldots \\
\ldots\end{array}$ & $\begin{array}{l}\ldots \\
\ldots\end{array}$ & $\begin{array}{l}\ldots \\
\ldots\end{array}$ & $\begin{array}{l}\ldots \\
\ldots\end{array}$ & $\ldots$ \\
\hline
\end{tabular}

Abbreviations as in table III.

The MICs for miconazole (table V) were more difficult to determine. Endpoints were less reproducible and the values shown are the highest obtained after three tests of the difficult isolates. One hundred and thirty-four determinations were repeated, with final MICs ranging from 4 to $25 \mu \mathrm{g} / \mathrm{ml}$. All the isolates were sensitive in the range $0 \cdot 1-40 \mu \mathrm{g} / \mathrm{ml}$, the highest MICs being obtained from $T$. glabrata and C. tropicalis.

The MICs for ketoconazole (table VI) were also difficult to determine. In 76 tests on C. albicans, growth was not present at one concentration only to reappear at a higher concentration; this effect was reproducible for 73 of the 76 isolates. The MIC in these cases was taken as the highest concentration that 
TABLE VI

Minimum inhibitory concentration of ketoconazole for yeast isolates

\begin{tabular}{|c|c|c|c|c|c|c|c|c|c|c|c|c|c|c|c|}
\hline \multirow[b]{2}{*}{ Species } & \multirow{2}{*}{$\begin{array}{c}\text { Group } \\
\text { derivation }\end{array}$} & \multirow{2}{*}{$\begin{array}{c}\text { Number } \\
\text { tested }\end{array}$} & \multicolumn{13}{|c|}{$\begin{array}{l}\text { Number of isolates inhibited by ketoconazole at } \\
\text { concentrations }(\mu \mathrm{g} / \mathrm{ml}) \text { of }\end{array}$} \\
\hline & & & $0 \cdot 1$ & 0.5 & $1 \cdot 0$ & 2.0 & $4 \cdot 0$ & $5 \cdot 0$ & 10 & 15 & 20 & 25 & 30 & 40 & 50 \\
\hline C. albicans & $\begin{array}{l}\mathrm{O} \\
\mathrm{L}\end{array}$ & $\begin{array}{l}671 \\
256\end{array}$ & $\begin{array}{l}\cdots \\
\cdots\end{array}$ & $\begin{array}{l}1 \\
1\end{array}$ & $\begin{array}{l}\ldots \\
\ldots\end{array}$ & $\begin{array}{l}5 \\
2\end{array}$ & $\begin{array}{l}28 \\
\cdots\end{array}$ & $\begin{array}{r}24 \\
3\end{array}$ & $\begin{array}{r}111 \\
87\end{array}$ & $\begin{array}{l}87 \\
93\end{array}$ & $\begin{array}{r}123 \\
41\end{array}$ & $\begin{array}{r}143 \\
21\end{array}$ & $\begin{array}{r}106 \\
8\end{array}$ & $\begin{array}{l}43 \\
\ldots\end{array}$ & $\begin{array}{l}\cdots \\
\cdots\end{array}$ \\
\hline C. tropicalis & $\stackrel{\mathrm{O}}{\mathrm{L}}$ & $\begin{array}{r}164 \\
74\end{array}$ & $\dddot{1}$ & $\dddot{i}$ & $\dddot{2}$ & $\dddot{i}$ & $\begin{array}{l}5 \\
1\end{array}$ & $\dddot{1}$ & $\begin{array}{l}42 \\
\ldots\end{array}$ & $\begin{array}{r}5 \\
16\end{array}$ & $\begin{array}{l}78 \\
10\end{array}$ & $\begin{array}{l}11 \\
31\end{array}$ & $\begin{array}{r}23 \\
7\end{array}$ & $\dddot{3}$ & $\begin{array}{l}\ldots \\
\cdots\end{array}$ \\
\hline C. pseudotropicalis & $\begin{array}{l}\mathrm{O} \\
\mathrm{L}\end{array}$ & $\begin{array}{r}29 \\
7\end{array}$ & $\begin{array}{l}\ldots \\
\ldots\end{array}$ & $\begin{array}{l}\ldots \\
\cdots\end{array}$ & $\begin{array}{l}8 \\
1\end{array}$ & $\begin{array}{l}\cdots \\
\cdots\end{array}$ & $\begin{array}{l}1 \\
2\end{array}$ & $\begin{array}{c}1 \\
\ldots\end{array}$ & $\dddot{\ldots}$ & $\begin{array}{r}4 \\
\ldots\end{array}$ & $\begin{array}{l}7 \\
2\end{array}$ & $\begin{array}{c}4 \\
\ldots\end{array}$ & $\begin{array}{r}4 \\
\ldots\end{array}$ & $\begin{array}{l}\ldots \\
\ldots\end{array}$ & $\begin{array}{l}\ldots \\
\ldots\end{array}$ \\
\hline C. guilliermondi & $\begin{array}{l}\mathrm{O} \\
\mathrm{L}\end{array}$ & $\begin{array}{l}14 \\
18\end{array}$ & $\ldots$ & $\begin{array}{r}1 \\
\ldots\end{array}$ & $\dddot{4}$ & $\dddot{6}$ & $\begin{array}{l}2 \\
2\end{array}$ & $\dddot{6}$ & $\begin{array}{l}\ldots \\
\ldots\end{array}$ & $\begin{array}{l}11 \\
\ldots\end{array}$ & $\begin{array}{l}\ldots \\
\ldots\end{array}$ & $\begin{array}{l}\ldots \\
\ldots\end{array}$ & $\begin{array}{l}\ldots \\
\ldots\end{array}$ & $\begin{array}{l}\ldots \\
\ldots\end{array}$ & $\begin{array}{l}\ldots \\
\ldots\end{array}$ \\
\hline C. parapsilosis & $\begin{array}{l}\mathrm{O} \\
\mathrm{L}\end{array}$ & $\begin{array}{r}11 \\
8\end{array}$ & $\ldots$ & $\begin{array}{l}\ldots \\
\ldots\end{array}$ & $\begin{array}{l}\ldots \\
\ldots\end{array}$ & $\begin{array}{l}\ldots \\
\ldots\end{array}$ & $\begin{array}{l}\ldots \\
\ldots\end{array}$ & $\begin{array}{l}\ldots \\
\ldots\end{array}$ & $\begin{array}{l}\ldots \\
\ldots\end{array}$ & $\begin{array}{l}\ldots \\
\ldots\end{array}$ & 2 & $\begin{array}{l}4 \\
3\end{array}$ & $\begin{array}{l}4 \\
2\end{array}$ & $\begin{array}{l}3 \\
1\end{array}$ & $\begin{array}{l}\ldots \\
\ldots\end{array}$ \\
\hline C. krusei & $\begin{array}{l}\mathrm{O} \\
\mathrm{L}\end{array}$ & $\begin{array}{r}21 \\
0\end{array}$ & $\ldots$ & $\begin{array}{l}\ldots \\
\ldots\end{array}$ & $\begin{array}{l}\ldots \\
\ldots\end{array}$ & $\begin{array}{l}\ldots \\
\ldots\end{array}$ & $\begin{array}{r}1 \\
\ldots\end{array}$ & $\begin{array}{r}7 \\
\ldots\end{array}$ & $\begin{array}{l}\ldots \\
\ldots\end{array}$ & $\begin{array}{r}4 \\
\ldots\end{array}$ & $\begin{array}{r}3 \\
\ldots\end{array}$ & $\begin{array}{c}6 \\
\ldots\end{array}$ & $\begin{array}{l}\ldots \\
\ldots\end{array}$ & $\begin{array}{l} \\
\ldots \\
\ldots\end{array}$ & $\begin{array}{l}\ldots \\
\ldots\end{array}$ \\
\hline C. mycoderma & $\begin{array}{l}\mathrm{O} \\
\mathrm{L}\end{array}$ & $\begin{array}{r}23 \\
8\end{array}$ & $\ldots$ & $\begin{array}{l}\ldots \\
\ldots\end{array}$ & $\begin{array}{l}\ldots \\
\ldots\end{array}$ & $\begin{array}{l}\ldots \\
\ldots\end{array}$ & $\begin{array}{l}\ldots \\
\ldots\end{array}$ & $\dddot{i}$ & $\begin{array}{l}\ldots \\
\ldots\end{array}$ & $\begin{array}{l}8 \\
3\end{array}$ & $\begin{array}{l}7 \\
3\end{array}$ & $\begin{array}{l}4 \\
1\end{array}$ & $\begin{array}{r}2 \\
\ldots\end{array}$ & $\begin{array}{r}2 \\
\ldots\end{array}$ & $\begin{array}{l}\ldots \\
\ldots\end{array}$ \\
\hline T. glabrata & $\begin{array}{l}\mathrm{O} \\
\mathrm{L}\end{array}$ & $\begin{array}{l}24 \\
21\end{array}$ & $\begin{array}{l}\ldots \\
\ldots\end{array}$ & $\begin{array}{l}\ldots \\
\ldots\end{array}$ & $\dddot{3}$ & $\begin{array}{l}\ldots \\
\ldots\end{array}$ & $\begin{array}{l}\ldots \\
\ldots\end{array}$ & $\dddot{8}$ & $\begin{array}{l}\ldots \\
\ldots\end{array}$ & $\begin{array}{l}\ldots \\
\ldots\end{array}$ & $\begin{array}{l}\ldots \\
\ldots\end{array}$ & $\begin{array}{l}4 \\
8\end{array}$ & $\begin{array}{l}14 \\
\ldots\end{array}$ & $\cdots_{1}$ & $\begin{array}{l}6 \\
1\end{array}$ \\
\hline R. rubra & $\begin{array}{l}\mathrm{O} \\
\mathrm{L}\end{array}$ & $\begin{array}{r}19 \\
1\end{array}$ & $\begin{array}{l}\ldots \\
\ldots\end{array}$ & $\begin{array}{l}\ldots \\
\ldots\end{array}$ & $\begin{array}{l}\ldots \\
\ldots\end{array}$ & $\begin{array}{l}11 \\
\ldots\end{array}$ & $\begin{array}{c}4 \\
\ldots\end{array}$ & $\begin{array}{l}\ldots \\
\ldots\end{array}$ & $\begin{array}{l}\cdots \\
\ldots\end{array}$ & $\begin{array}{l}4 \\
1\end{array}$ & $\begin{array}{l}\ldots \\
\ldots\end{array}$ & $\begin{array}{l}\ldots \\
\ldots\end{array}$ & $\begin{array}{l}\ldots \\
\ldots\end{array}$ & $\begin{array}{l}\ldots \\
\ldots\end{array}$ & $\begin{array}{l}\ldots \\
\ldots\end{array}$ \\
\hline
\end{tabular}

Abbreviations as in table III.

inhibited growth. As a check, the test was repeated with ketonazole at three times the MIC, to ensure that no more growth was possible; in no case was further growth seen. All isolates were sensitive to ketoconazole in the range $0 \cdot 1-50 \mu \mathrm{g} / \mathrm{ml}$.

\section{Discussion}

The two groups of patients studied were those with an oral malignancy in whom irradiation would directly affect the oral mucous membranes as well as the overlying skin of the lesion itself, and those who were treated for laryngeal carcinoma in whom the skin and the lesions would be directly irradiated but the oral mucous membrane would receive only peripheral irradiation.

The oral flora of both groups was sampled with cottonwool swabs. This technique is at best semi-quantitative and compares unfavourably with the more accurate imprint technique of Arendorf and Walker (1980). However, it is the most commonly used clinical method for sampling these sites and it was found that many of the patients in both groups had yeasts in their oral cavity before radiation. These may have been part of the normal commensal flora or may have become established as a consequence of neoplastic disease. However, immediately after the start of therapy there was a rapid increase in the isolation of yeasts from all sites in both groups. This increase continued throughout treatment and persisted at least for 6 months beyond the treatment period. The reason for this prolonged increase is not known, because the oral 
mucosa in both groups appears clinically to return almost to normal after radiotherapy with no signs of fungal infection (Al-Tikriti, 1980). This reservoir of fungi could be a source for further oral or disseminated fungal infection, and in such debilitated patients must be considered a significant clinical hazard. A similar increase in fungal isolations was seen at the skin sites of irradiation in both groups; it persisted for at least 6 months in the oral group but showed slight evidence of diminution at this time in the laryngeal group. The increased isolation of fungi was coincident with a predominantly moist desquamation of the skin surface of the type described by Moss et al. (1973). The fungi isolated from the skin were probably responsible wholly or in part for the reactions seen. It is also possible that the radiation had altered the normal barrier properties of the skin and its secretions, making it more susceptible to infection. These latter effects of radiation have been noted previously (Walter et al., 1979) but the possibility that they could be exacerbated by fungi has not.

It is interesting to speculate on the source of the fungi found on the skin after the start of radiation because these organisms are usually confined to moist skin folds in the non-debilitated patient (Somerville, 1969; Odds, 1979). One possible source is the mouth, from which the fungi might be spread on the fingers; many of the oral isolates of C. albicans had MICs identical to those of isolates from the skin. However, to establish such a link firmly it would be necessary to type oral and skin strains by a suitable method, for example that of Warnock et al. (1979).

C. albicans was the most numerous isolate, confirming the results of Rosenthal and Wilkie (1965) and Bodey (1975). The increase in growth of this organism was found in both groups of patients despite the fact that the laryngeal group had not orally directed irradiation. One explanation for this increase may be that peripheral radiation affected the submandibular and parotid salivary glands, causing a change in the composition and quality of saliva (Al-Tikriti, 1980); such a change of salivary-gland function and increase in yeast infection was noted by Robinson (1976), who suggested that the antimicrobial substances in saliva regulate the number of yeasts in the mouth.

In addition to C. albicans, six other species of Candida were isolated, of which $C$. tropicalis was the most numerous. This latter organism is often isolated from clinical specimens, accounting for $18 \%$ of laboratory growths in one survey (Walker and Luecke, 1974). The isolation of $T$. glabrata and $R$. rubra from oral sites was noted by Budtz-Jorgensen, Stenderup and Grabowski (1975). However, the significance of these organisms in mixed infections is not known. Similarly, the significance of Klebsiella spp. is not known, although these organisms are often isolated from patients with neoplastic disease (Singer, Kaplan and Armstrong, 1977). The isolation of Klebsiella did not follow any definite pattern or distribution among the patients sampled, this result being in agreement with the work of Holbrook and Russell (1979) and Martin (1981). Klebsiella probably becomes established as a transitory opportunist.

Organisms resistant to any of the antifungal agents were not found. The 
MICs for most of the species tested were considerably higher than those recorded by Bannatyne and Cheung (1978) and Moody et al. (1980), but were similar to those of Dixon et al. (1978) and Odds et al. (1980). The in-vitro tests suggest that any of the antifungal drugs studied would be appropriate for treatment of the superficial fungal infections seen in these patients. Because the isolates tested were selected on the basis of colonial morphology, resistant strains may not have been included; however, the chances of this would have been decreased by the large number of isolates tested.

The MICs of nystatin and amphotericin B were low, except against $C$. tropicalis and C. krusei; this is in agreement with the results of Athar and Winner (1971).

However, on 23 occasions, the MIC of nystatin against $C$. albicans isolates from the same site differed by at least threefold. This may be evidence that two distinct strains of $C$. albicans had infected the same site. However, it will be necessary to examine these strains in more detail before such an assertion can be proved.

The MICs for miconazole and ketoconazole were not in agreement with those reported by Bannatyne and Cheung (1978) and Moody et al. (1980). Moody et al. (1980) suggested that their low MIC values for yeasts were a consequence of their use of fresh isolates, which may be more active metabolically. In our study fresh isolates have been used and therefore this explanation is probably not valid. The difference between our results and those of Moody et al. (1980) could reflect the different methods used. We used a heavy inoculum of approximately $1 \times 10^{6} \mathrm{cells} / \mathrm{ml}$; initial studies (unpublished) had shown that this gave reproducible results. Despite the heavy inoculum, there were difficulties in obtaining MIC values for the imidazoles, particularly ketoconazole. Apparent disappearance and re-emergence of growth at higher concentrations of the drug were found, for which an explanation is still lacking. The lower MIC results for ketoconazole obtained by Moody et al. (1980) may have been a result of recording the first disappearance of growth rather than the second. The use of ketoconazole for the treatment of fungal infections is likely to increase because the preliminary tests have given encouraging results in animals (Odds et al., 1980).

Our results show that many fungi can be isolated from the oral and skin sites of irradiation in patients receiving radiotherapy. This increase persists for a prolonged period after treatment. It remains to be determined which therapeutic regimes can significantly suppress the fungal flora during and after treatment. It is to be hoped that the treatment of these fungal infections will ameliorate some of the distressing effects of radiation experienced by these patients.

We thank Dr G. M. King and his colleagues at Weston Park Hospital, Sheffield for their help and co-operation, and the Iraqi Ministry of Higher Education for their financial support of U. Al-T.

\section{REFERENCES}

AL-TIKRITI, U. 1980. The clinical and microbiological changes in oral mucosa secondary to radiotherapy. M.Med.Sci. thesis, University of Sheffield. 
ARENDORF, T. M. AND WALKER, D. M. 1980. The prevalence and intra-oral distribution of Candida albicans in man. Archs. oral Biol., 25, 1.

Athar, M. A. AND WinNer, H. I. 1971. The development of resistance by Candida species to polyene antibiotics in vitro. J. med. Microbiol., 4, 505.

Bannatyne, R. M. AND Cheung, R. 1978. Susceptibility of Candida albicans to miconazole. Antimicrob. Agents Chemother. 13, 1040.

Beumer, J., Curtis, T. AND Harrison, R. E. 1979. Radiation therapy of the oral cavity: sequelae and management. 1. Head and Neck Surg., 1, 301.

BoDEY, G. P. 1975. Infections in cancer patients. Cancer Treat. Rev., 2, 89.

BoggS, D. R., Williams, A. F. AND Howell, A. 1961. Thrush in malignant neoplastic disease. Archs intern. Med., 107, 354.

BudTZ-Jorgensen, E., STENDERUP, A. AND GRABOwSKI, M. 1975. An epidemiologic study of yeasts in elderly denture wearers. Commun. dent. oral Epidem., 3, 115.

Сно, S. Y. AND Chol, H. Y. 1979. Opportunistic fungal infection among cancer patients. A 10 year autopsy study. Am. J. clin. Path., 72, 617.

Cowan, S. T. 1974. Cowan and Steel's Manual for the identification of medical bacterial, 2nd ed., Cambridge University Press, Cambridge, p. 111.

Dixon, D., Shadomy, S., Shadomy, H. J., Espinel-Ingroff, A. ANd Kerkering, T. M. 1978. Comparison of the in vitro antifungal activities of miconazole and a new imidazole, R41,400. J. infect. Dis., 138, 245.

Dolan, C. T. 1971. A practical approach to the identification of yeast-like organisms. Am.J. clin. Path., 55, 580.

HolbrooK, W. P. and Russell, C. 1979. Klebsiella in patients with denture stomatitis. $B r$. dent. J., 146, 3.

MARTIN, M. V. 1981. Isolation of oral bacteria. Br. dent. J., 150, 85.

Martin, M. V. AND Schneidau, J. D. 1970. A simple and reliable assmilation test for the identification of Candida species. Am. J. clin. Path., 53, 875.

MoOdY, M. R., Young, V. M., MoRrIS, M. J. AND SCHIMPFF, S. C. 1980 . In vitro activities of miconazole, miconazole nitrate and ketoconazole alone and combined with rifampin against Candida spp. and Torulopsis glabrata recovered from cancer patients. Antimicrob. Agents Chemother., 17, 871.

Moss, W. T., Brand, W. N. AND Battifora, H. 1973. Radiation oncology; rationale, technique, results, 4th ed., C.V. Mosby, St Louis, pp. 53-146.

ODDs, F. C. 1979. Candida and candidosis, Leicester University Press, Leicester, p. 81.

Odds, F. C., Milne, L. J. R., Gentles, J. C. ANd Ball, E. H. 1980. The activity in vitro and in vivo of a new imidazole antifungal ketoconazole. J. antimicrob. Chemother., 6, 97.

RoBInson, J. E. 1976. Characteristics of irradiated soft and hard tissues. J.prosth. Dent., 35, 549.

Rosenthal, L. E. AND WiLkIE, B. 1965. The effects of radiotherapy on oral tissues. J. prosth. Dent., 15, 153.

SHADOMY, S. 1969. In vitro studies with 5-fluorocytosine. Appl. Microbiol, 17, 871.

Shannon, I. L., Trodahl, J.N. AND StarCKe, E. N. 1978. Remineralisation of enamel by a saliva substitute designed for use by irradiated patients. Cancer, 41, 1746.

ShIPMAN, B. 1979. Clinical evaluation of oral Candida in cancer chemotherapy patients. $J$. prosth. Dent., 41, 63.

SingER, C., KAPLAN, M. H. AND ARMSTRONG, D. 1977. Bacteremia and fungemia complicating neoplastic disease: a study of 364 cases. Am. J. Med., 62, 731 .

SOMER VILLE, D. A. 1969. The normal flora of the skin in different age groups. Br.J.Derm., 81, 248.

WALKER, L. J. AND LUECKE, M. R. 1974. The identification of yeasts from clinical material. Proc. Iowa Acad. Sci., 81, 14.

Walter, J. B., Miller, H. AND Bomford, C. K. 1979. A short textbook of radiotherapy, 4th rev. ed., Churchill Livingstone, Edinburgh.

Warnock, D. W., SPEller, D. C. E., DAy, J. K. AND Farrell, A. J. 1979. Resistogram method for the differentiation of strains of Candida albicans. J. appl. Bact., 46, 571. 